\title{
Diversité des espèces végétales au sein des agro-forêts à base d'anacardier dans la commune de Savalou au Benin
}

\author{
Ibouraïma YABI*, Francis YABI BIAOU et Sévérin DADEGNON \\ Département de Géographie et Aménagement du Territoire (DGAT), \\ Université d'Abomey-Calavi (UAC), Benin. \\ *Auteur correspondant, E-mail : yafid2@yahoo.fr
}

\section{RESUME}

Cette étude sur la diversité des espèces végétales des agro-forêts à base de l'anacardier vise essentiellement la connaissance des espèces végétales ligneuses compagnes à Anacardium occidentale dans la commune de Savalou. Pour ce faire, ces agro-forêts ont été étudiées en y effectuant un inventaire floristique à travers 157 placettes installées. Les informations ainsi obtenues ont été traitées par une analyse de la diversité alpha et Bêta. L'approche méthodologique adoptée a permis de recenser, 37 espèces ligneuses regroupées dans 18 familles dans les agro-forêts de la commune de Savalou. Les familles les plus représentées sont les Caesalpiniaceae (5 espèces). En outre, le regroupement en trois zones (Ouest, Centre et Est) des espèces a permis de montrer que l'indice de diversité de Shannon varie de 1,6 bit à 2,48 bits alors que l'équitabilité de Pielou oscille entre 0,34 à 0,51 . Les solutions envisagées pour une gestion durable des agro-forêts basées sur l'anacardier concernent les modes d'accès et de valorisation de la la terre agricole et la nécessité de la conservation de la biodiversité.

(C) 2013 International Formulae Group. All rights reserved.

Mots clés: Commune de Savalou, agro-forêts à base d'anacardier, diversité biologique, gestion.

\section{INTRODUCTION}

Dans les pays en voie de développement, plus particulièrement au Bénin, l'agriculture demeure le socle de l'économie nationale. Le secteur industriel étant encore embryonnaire, l'agriculture emploie $75 \%$ des actifs et réalise $85 \%$ des recettes d'exportation d'origine intérieure (Zoumènou, 2007). Pour satisfaire ses besoins nutritionnels, l'Homme intègre dans son alimentation les fruits (Aïchéou, 2011). L'anacardier (Anacardium occidentale) occupe une place prépondérante dans l'économie nationale (Atchadé, 2004). Une étude de la FAO (2002) estime la surface couverte par cette culture au Bénin entre 60 à 70.000 ha répartis sur six des douze départements du pays. Le Bénin se hisse ainsi parmi les dix premiers producteurs mondiaux avec $2 \%$ de la production. En 2004, les exportations de noix brutes ont atteint 50.000 tonnes alors qu'elles n'étaient en 1996 que de l'ordre de 10.000 tonnes, soit une multiplication par 5 en 8 ans (Tandjiékpon, 2005). Cela témoigne du rôle important que joue la filière anacarde sur l'économie 
nationale du Bénin en générale et sur les ménages agricoles de la commune de Savalou en particulier. S'agissant de la superficie emblavée par la production de l'anacarde, une étude du Centre Communal pour la Promotion Agricole (CeCPA) de Savalou en 2011 a fait état de 11.507 ha de plantations appartenant à 2884 producteurs. De ce fait, l'anacardier est qualifié dans le secteur d'étude de "cacao du Bénin" (Yabi, 2004) par analogie à la production du cacao en Côte d'Ivoire. Il joue un rôle prépondérant sur le plan économique, écologique et culturel. Le rôle de l'anacardier sur le plan culturel n'est plus à démontrer car à la fin de chaque campagne de commercialisation du produit, son économie sert à organiser les cérémonies. De même sur le plan infrastructurel, il sert également à l'acquisition de quelques biens et services comme la construction des infrastructures, l'achat des matériels roulants.

Sur le plan écologique, Tandjiékpon (2005) et Djogbénou (2007) ont montré que l'agroforesterie, surtout celle à base de l'anacardier développée par les populations paysannes du département des Collines est l'une des solutions les plus adéquates pour pallier aux effets de dégradation de l'environnement. Dans ce cadre, elle a un impact positif sur la dynamique de l'occupation du sol et présente un intérêt écologique dans le secteur d'étude. Au Bénin, le système agroforestier traditionnel est caractérisé par la présence d'espèces naturelles telles que Vitellaria paradoxa (karité), Parkia biglobosa (néré), Adansonia digitata (baobab), Tamarindus indica (tamarinier), Afzelia africana (lingué) au Nord tandis que dans la partie Sud, les espèces ligneuses comme Elaeis guineensis (palmier), Irvingia gabonensis (pommier), Triplochiton scleroxylon (samba), Milicia excelssa (iroko), prédominent (Sokpon, 1994). Ces essences sont épargnés lors des défrichements en raison de leurs utilités socioéconomiques (Wala et al., 2005). Mais, Yabi et al., (2009) ont constaté que de plus en plus, le paysage agraire dans le centre du Bénin est caractérisé par une association de cultures annuelles sous plantation d'anacardiers (Anacardium occidentale). Cette nouvelle forme d'agroforesterie permet aux producteurs non seulement de lutter contre l'insécurité alimentaire mais également d'obtenir des gains monétaires complémentaires issus de la vente des noix d'anacarde (Yabi et al., 2012).

Mais, au-delà des intérêts socioéconomiques, l'agroforesterie à base de l'anacardier, tout comme les autres types, offre également des avantages écologiques dans la mesure où elle participe au reboisement des espaces agricoles et freine l'itinérance des producteurs à la recherche de nouvelles terres (Yabi et al., 2012). En outre, les arbres d'anacardiers cohabitent avec d'autres espèces végétales naturelles ou plantées. Les agro-forêts à base d'anacardier paraissent donc plus conservatrices de la biodiversité végétale.

Il est donc important d'étudier la diversité végétale au sein de ces écosystèmes afin de mieux appréhender leur pouvoir conservateur de la biodiversité végétale. D'un point de vue scientifique, la connaissance des espèces végétales compagnes de l'anacardier et $\mathrm{du}$ fonctionnement de ces agro-forêts, constitue un nouvel axe de recherche à explorer au regard de l'importance et de propagation rapide de cette espèce au Bénin. C'est pourquoi la présente étude a été initiée pour la réalisation de l'étude de référence sur la diversité biologique végétale des agroforêts à base d'anacardier dans la commune de Savalou, afin de poser les bases de la gestion durable des ces écosystèmes particuliers. Le point cardinal de cette recherche est d'apprécier la diversité des espèces végétales évoluant dans les agro-forêts à base d'anacardier.

\section{Milieu d'étude}

Le champ d'étude a concerné les plantations d'anacardier de la commune de Savalou (Figure 1). En effet, située dans le 
département des Collines au Bénin, la commune de Savalou est comprise entre $7^{\circ} 35^{\prime}$ et $8^{\circ} 13^{\prime}$ de latitude nord et entre $1^{\circ} 30^{\prime}$ et $2^{\circ} 60^{\prime}$ de longitude est. Elle est limitée à l'est par les communes de Dassa-Zoumè et de Glazoué, à l'ouest par la République du Togo sur environ $65 \mathrm{~km}$, au sud par la commune de Djidja et au nord par celle de Bantè. Elle couvre une superficie de $2674 \mathrm{~km}^{2}$, soit 2,37\% du territoire national.

La température moyenne annuelle dans ce secteur est de $27,35{ }^{\circ} \mathrm{C}$. Sur la base de la répartition des précipitations, on distingue une saison pluvieuse de mars à octobre et une saison sèche de novembre à février. Les moyennes annuelles de la pluviométrie est de 1124, $74 \mathrm{~mm}$. Ce qui permet d'avoir une disponibilité en eau au sol nécessaire pour le développement et la croissance de l'espèce Anacardium occidentale dans la commune de Savalou. Le relief est constitué d'une pénéplaine de $300 \mathrm{~m}$ d'altitude en moyenne. Cette pénéplaine est parsemée de collines dont l'altitude atteint $520 \mathrm{~m}$ avec des pentes variant entre 3 et $10 \%$ dans les sites agglomérés. C'est une plaine érodée qui s'est développée sur du gneiss et laissant en relief les éléments granitiques ou des carapaces ferrugineuses les plus résistantes. D'après les travaux de Akoègninou (2004), le milieu naturel de la commune de Savalou est composé des îlots de forêts denses, de savanes, de jachères et de champs. Le domaine des forêts denses a été transformé en plantations (agro-forêts), en vieilles jachères et en champs cultivés.

\section{Méthode d'étude \\ Collecte des données}

Le recensement des espèces végétales est fait suivant le zonage (Est, Centre et Ouest) délimité par le centre communal pour la promotion agricole dans la commune de Savalou. Un total de 157 placettes circulaires de $12 \mathrm{~m}$ de rayon ont été installées (Figure 2). La méthode stigmatiste de Braun-Blanquet (1932) est utilisée pour la collecte des données phytosociologiques. A l'intérieure de chaque placette, les espèces végétales ligneuses ont été inventoriées et affectées de coefficients d'abondance-dominance. L'échelle d'abondance dominance utilisée est celle de BraunBlanquet (1932). Les espèces ont été identifiées directement sur le terrain grâce à la Flore du Bénin (Akoègninou et al., 2006). Les espèces indéterminées sont herborisées et identifiées à l'herbier National du Bénin.

\section{Analyse des données}

\section{Analyse des données de relevés phytosociologiques}

Cette analyse prend en compte la diversité Alpha (l'indice de diversité de Shannon et l'équitabilité de Piélou) et la diversité Bêta par le biais de l'indice de Jaccard.

- $\quad$ Richesse spécifique (S)

Elle est déterminée par le nombre total d'espèces enregistrées par groupement végétal.

- Indice de diversité de Shannon-Wienner (H) (Shannon, 1948)

Il est déterminé par la formule :

$$
H=-\sum_{i}^{n} P i \log _{\mathbf{z}} P i
$$

avec $\mathrm{P}_{\mathrm{i}}=\mathrm{r}_{\mathrm{i}} / \mathrm{r}$; où $\mathrm{r}_{\mathrm{i}}$ est le recouvrement de l'espèce $\mathrm{i}$ dans le relevé considéré et $r$ désigne la somme totale des recouvrements des espèces du relevé. $H$ s'exprime en bits. Il varie généralement entre 1 et 5 bits.

Lorsque $\mathrm{H}$ est élevé $(\mathrm{H}>3,5)$ cela signifie une forte diversité au sein du groupement végétal ce qui traduit que les conditions de la station sont très favorables à l'installation d'un grand nombre d'espèces dans des proportions quasi-égales. Par contre si $\mathrm{H}$ est faible $(\mathrm{H}<2,6)$ cela signifie que les conditions du milieu sont très défavorables et induisent une forte spécialisation des espèces ; on a alors un groupement dominé par quelques espèces qui se partagent en grande partie le recouvrement au niveau du groupement.

- $\quad$ Equitabilité de Piélou (E) (Pielou, 1969) 
Elle traduit le degré de diversité atteint par rapport au maximum théorique (Blondel, 1979). Il est calculé par la formule :

$$
E=\frac{H}{H \max }
$$

avec $\mathrm{H}_{\max }=\log 2(\mathrm{~S}), \mathrm{S}$ désignant la richesse spécifique. Elle est comprise entre 0 et 1 . E tend vers 0 lorsque la quasi-totalité des individus appartiennent à une seule espèce et prend la valeur 1 lorsque toutes les espèces ont exactement le même recouvrement.

- $\quad$ Indice de Jaccard (Ij)

Il traduit la similarité qui existe entre les groupements végétaux et se définit comme suit:

$\mathrm{Ij}=\frac{C}{A+B-C}$

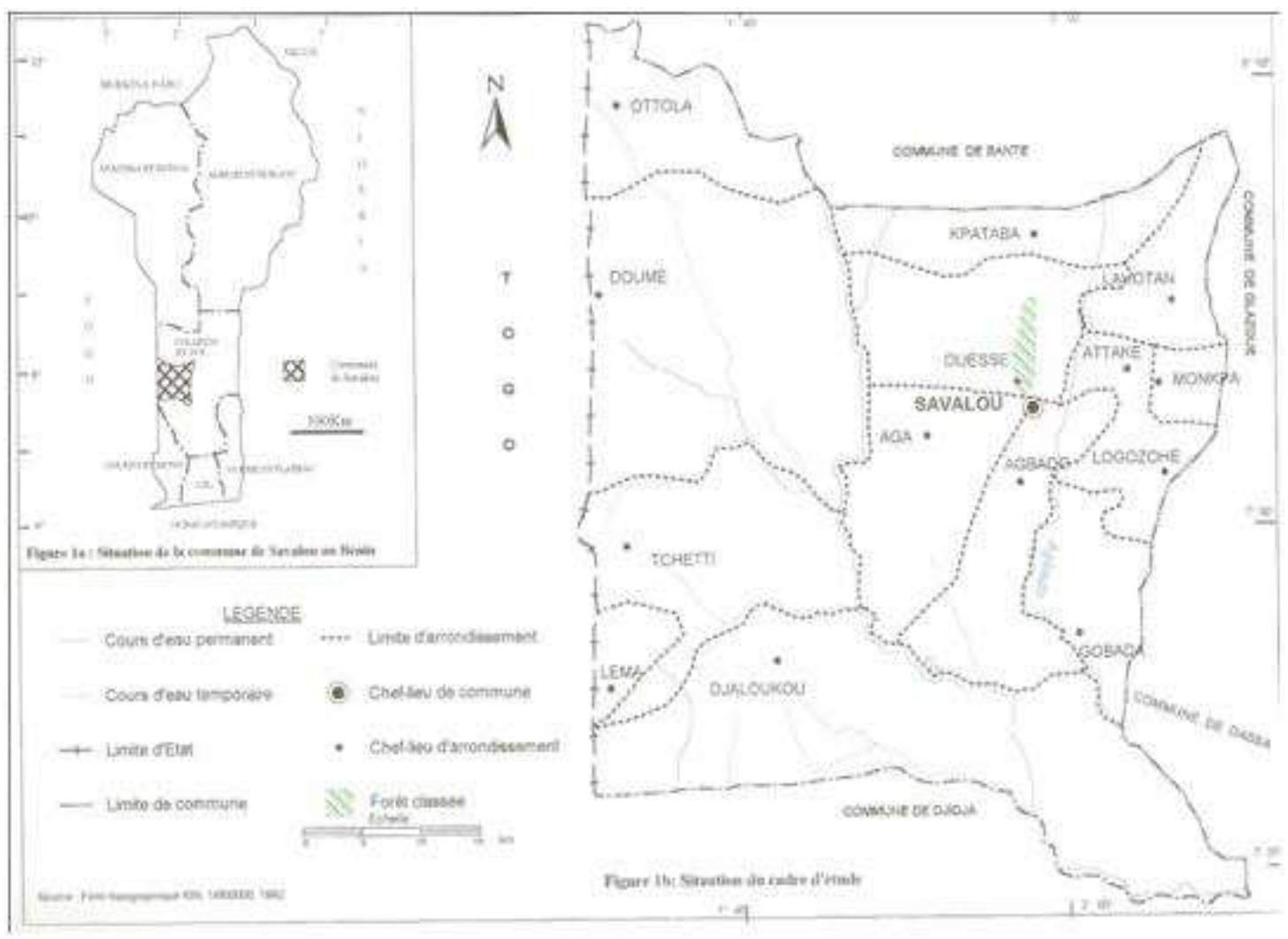

Figure 1: Carte de situation du milieu d'étude.
A désigne l'ensemble des espèces du groupement $\mathrm{A}$;

$\mathrm{B}$ désigne l'ensemble des espèces du groupement B ; groupements en comparaison. que les placettes des deux groupements sont différentes et ne partagent pas beaucoup d'espèces en commun. que les communautés végétales sont les mêmes.
$\mathrm{C}$ désigne les espèces communes aux deux

- Si Ij est inférieur à $50 \%$ on conclut

- Si Ij est supérieur à $50 \%$ on conclut 


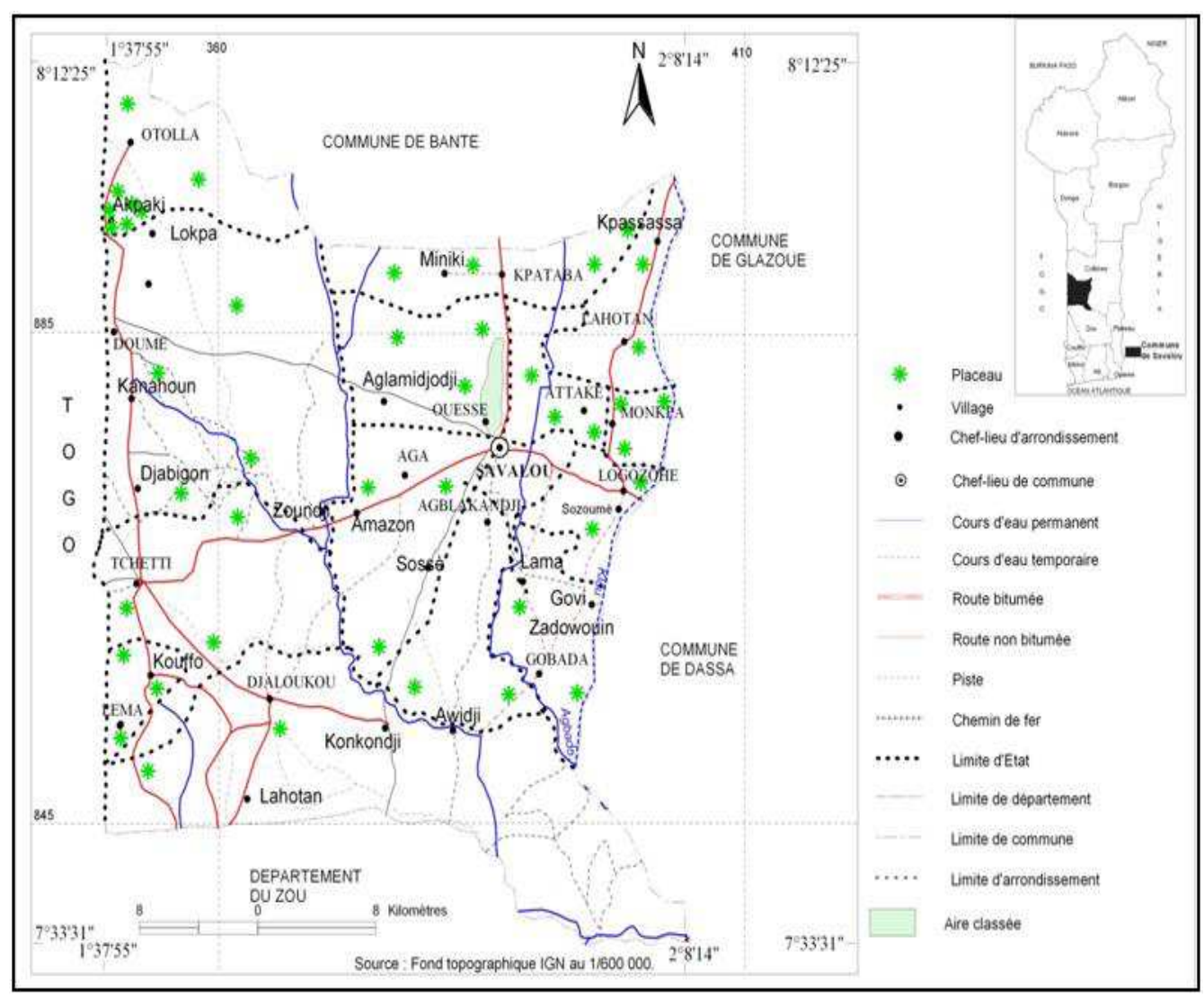

Figure 2: Carte de la répartition des placettes dans les agro-forêts de la commune de Savalou.

\section{RESULTATS}

\section{Analyse de la flore}

L'analyse de la flore des agro-forêts a porté sur la composition floristique d'une part puis, sur la diversité spécifique et la comparaison des groupements végétaux d'autre part.

\section{Composition et richesse spécifique des espèces végétales des agro-forêts}

L'analyse de la composition floristique montre que 37 espèces appartenant à 18 familles sont recensées dans les agro-forêts de la commune de Savalou (Figure 3). Le nombre d'espèces par famille varie de 1 à 5 . Les espèces Caesalpiniaceae sont représentées par 5 espèces, suivie des Mimosaceae et Anacardiaceae (4 espèces), des Verbanaceae, des Moraceae, des Meliaceae, et des combretaceae (3 espèces), des Fabaceae (2 espèces). Les dix autres familles (Arecaceae, Bignoniaceae, Bombacaceae, Celastraceae, Chrysobalanaceae, Euphorbiaceae,

Ochnaceae, Polygonaceae, Rutaceae, Sapotaceae) sont représentées chacune par une seule espèce.

\section{Diversité spécifique des formations végétales agro-forestières}

La diversité spécifique a permis d'apprécier la richesse biologique végétale des agrosystèmes au sein desquels évolue l'espèce Anacardium occidentale. Il faut souligner que les espèces ont été regroupées par zone (Zone Ouest, Zone Centre et Zone Est) selon la répartition définie par le CeCPA. Ainsi, la richesse spécifique, la diversité de Shannon (H) et l'indice d'équitabilité de Piélou (E) de chaque zone (Tableau 1) ont été calculés. 
L'indice de diversité de Shannon varie de 1,6 bit (zone Ouest) à 2,48 bits (zone Centre) alors que l'équitabilité de Pielou oscille entre 0,34 à 0,51. En outre, l'examen du Tableau 1 montre que la zone Est a une richesse spécifique plus élevée (31 espèces) et la zone Ouest sort la moins diversifiée avec 24 espèces. Il faut signaler que quelle que soit la zone considérée, l'indice de diversité de Shannon et l'équitabilité de Pielou sont faibles. Ces faibles valeurs obtenues traduisent une dominance en recouvrement de quelques espèces dans le milieu. Il s'agit notamment de l'espèce Anacardium occidentale. Néanmoins, la zone Centre et la zone Est se révèlent les plus diversifiées respectivement avec 2,48 et 2,31 bits que la zone Ouest (1,6 bit). La valeur plus élevée des indices de diversité dans les zones Est et Centre peut s'expliquer par le fait que les paysans n'entretiennent pas correctement les plantations d'anacardier, ce qui permet aux autres plantes de rentrer en compétition avec l'espèce Anacardium occidentale. L'indice de diversité de Shannon dans la zone Ouest est faible parce que les paysans dans ce milieu entretiennent la plantation d'Ancardium occidentale. Ce caractère fait qu'à chaque nettoyage, les espèces végétales en régénération sont détruites au profit de l'espèce Anacardium occidentale. Les quelques espèces végétales pérennes sont épargnées dans les champs du fait de leurs valeurs économiques. Ces espèces sont donc Vitellaria paradoxa, Parkia biglobosa et Pterocarpus erinaceus (Djèdji, 2011).

\section{Comparaison des communautés végétales selon les zones de production}

Les valeurs d'Indice de Jaccard (Ij) issues de la combinaison des placettes sont présentées dans le Tableau 2. De l'analyse de ce tableau on retient que quel que soit le type de zone considérée, le degré de similarité dépasse $50 \%$. On remarque que les placettes de la zone Ouest (Ottola, Tchetti, Lema, Doumè et Djalloukou) présentent une grande affinité avec les placettes de la zone Centre (Kpataba, Atakè, Ouèssè, Aga et Agbado). Ces grandes similarités résident dans le fait que les placettes de la zone Ouest ont les espèces telles que Afzelia africana, Albizia lebbeck, Azadirachta indica, Daniellia oliveri, Elaeis guineensis, Isoberlinia doka, Ficus spp, Mangifera indica, Parkia biglobosa, Pterocarpa erinaceus en commun avec les deux autres milieux. De même, il y a une grande similarité entre les placettes de la zone Centre et les placettes de la zone Est (Gobada, Logozohè, Monkpa et Lahotan), du fait des espèces Lannea acida, Hymenocardia acida, Pseudocedrela kotschyi, Delonix regia, Maytenus senegalensis, Milicia excelsa.

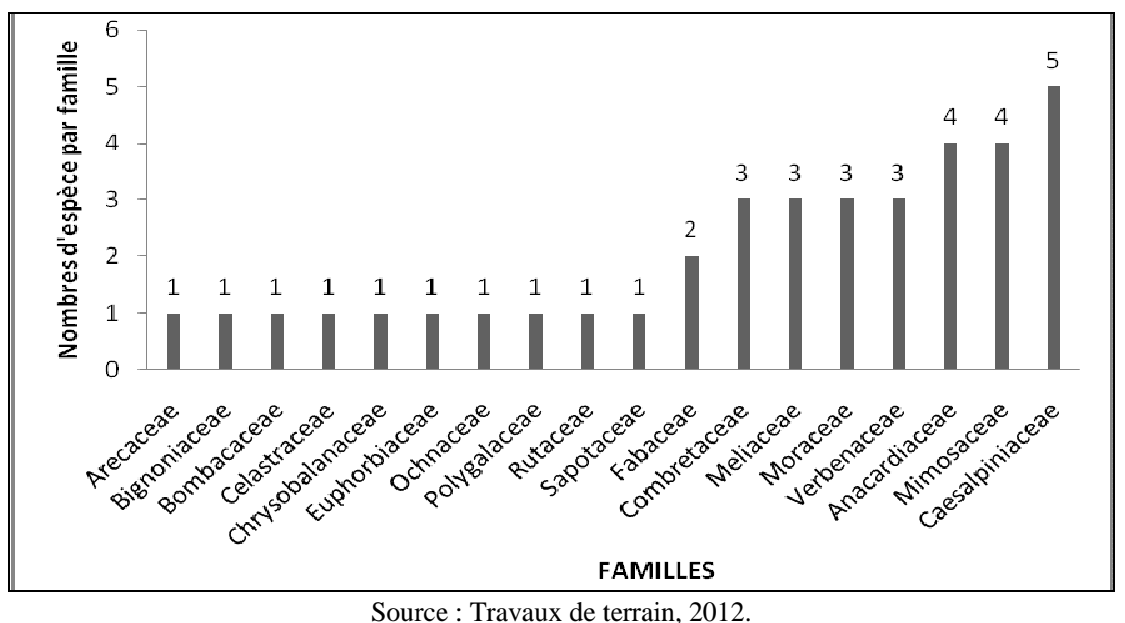

Figure 3: Nombre d'espèces par famille. 
Tableau 1 : Diversité spécifique des formations végétales.

\begin{tabular}{lccc}
\hline & Richesse spécifique & Indice de Shannon & Equitabilité de Pielou \\
\hline Zone Ouest & 24 & 1,6 & 0,34 \\
Zone Centre & 28 & 2,48 & 0,51 \\
Zone Est & 31 & 2,31 & 0,46 \\
\hline \multicolumn{2}{r}{ Source : Travaux de terrain, 2012.} & &
\end{tabular}

Tableau 2 : Indice de Jaccard en pourcentage des placeaux.

\begin{tabular}{lccc}
\hline Zones & Ouest / Centre & Ouest / Est & Centre / Est \\
\hline Indice de Jacard $(\%)$ & 62,50 & 61,80 & 64 \\
\hline
\end{tabular}

Source : Travaux de terrain, 2012

\section{DISCUSSION}

\section{Importance et caractéristiques des espèces végétales}

Dans la présente étude, 38 espèces ont été recensées et réparties en 19 familles. Les familles Anacardiacae et Leguminosae sont les plus représentées dans les agro-forêts avec respectivement $21,05 \%$ et $47,37 \%$ des espèces recensées. Cette recherche a mis en exergue les caractéristiques de diversité biologique végétale des agro-forêts d'anacardier dans la commune de Savalou. La valeur des indices de diversité de Shannon de chaque zone (Ouest, Centre et Est) est en dessous de la moyenne respectivement 1,$6 ; 2,48$ et 2,31 bits. Ces faibles valeurs obtenues peuvent s'expliquer par fait que ces milieux sont sujets aux pressions anthropiques. Ces résultats obtenus sont semblables à ceux obtenus par (Moguel et Toledo (1999) qui, aux termes de leurs études, ont constaté que la principale source de la diversité biologique végétale des systèmes agro-forestiers provient des espèces herbacées. Par contre, Soto-Pinto et al. (2000) ont émis une précision par rapport à ces faibles valeurs de diversité. Selon ces auteurs, si les pratiques à l'intérieur du système agroforestier s'intensifient ou si l'agriculteur ne permet que la croissance de quelques espèces en fonction de leur valeur économique, la biodiversité diminue. C'est pourquoi la rotation des cultures peut être nécessaire afin de permettre la régénération de certaines espèces (Okullo et Waithum, 2007). Ceci démontre bien le lien qui doit exister entre la préservation de l'intégrité des écosystèmes et les gains économiques individuels.

De ces constats, on peut donc déduire que pour améliorer la stabilité et la durabilité écologique des agro-forêts à base de l'anacardier, l'élagage ou l'ajout des espèces végétales indigènes est une solution envisageable pour accroître la diversité biologique. Une fois que cette mesure est respectée, la diversité au sein des agro-forêts à base de l'anacardier pourrait s'accroître. Les études réalisées par Daniele et Michael (2011) à Sulawesi dans la partie centrale de l'Indonésie ont révélé que dans les agro-forêts à base de Cacao, la diversité spécifique est relativement importante ( 91 espèces), même si elle est inférieurs à celle des deux écosystèmes forestiers naturels connexes (171 espèces). Dans le même temps, Bisseleua et al., (2008) ont recensée 260 espèces herbacées trouvées dans les agro-forêts à base de cacao au Cameroun. Par contre (Lott et al., 2000) ont démontré que la production primaire arborée totale est généralement supérieure en forêt qu'en système agro-forestier. En définitive, l'agroforesterie est plus durable, en termes de biodiversité que l'agriculture moderne. Il n'est donc pas totalement très juste d'affirmer cela surtout lorsque la comparaison est faite avec une forêt naturelle. Cette précision est capitale, parce qu'il ne faudrait pas que l'agroforesterie devienne un instrument pour la conversion des forêts primaires en systèmes agro forestiers. Toutefois, sachant que la perte d'habitats par 
déforestation est l'une des causes premières de la perte de biodiversité, l'agroforesterie pourrait être utilisée comme régulatrice afin de diminuer les effets négatifs de la dégradation des forêts (Dussault, 2008).

\section{Espèces pérennes et gestion des agro-forêts à base d'anacardier}

Les pratiques de l'agroforesterie à base de l'anacardier ne sont soumises à aucune norme en matière de protection de l'environnement. Dans la commune de Savalou, aucune mesure de protection des essences végétales n'est mise en œuvre avant l'installation des plantations d'anacardier. Même un simple inventaire des espèces végétales n'est entrepris pour préalablement identifier les essences de valeurs inscrites sur la liste rouge de l'UICN et/ou menacées d'extinction sur le plan national. Les techniques culturales dans le centre du Bénin qui consiste à éliminer toutes les espèces végétales avant l'installation de n'importe quelle culture n'est pas de nature à conserver les espèces de valeur (Photo1).

Il faut donc orienter les efforts vers les études d'impact environnemental pour prédire et corriger au fur et à mesure les effets néfastes de ces activités sur le milieu biophysique. De même, il très important de penser à l'initiation des formations ou sensibilisation pouvant amener les agriculteurs à se rappeler de la notion de conservation de la diversité biologique en général. En Indonésie par exemple, les études de Danielie et Michael (2011) ont prouvé que dans cette région du globe, les agriculteurs ont une certaine connaissance de la protection de l'environnement axée sur l'agroforesterie à base du Cacao. Dans ce pays, une marge de change est réservée aux espèces végétales qui peuvent cohabiter avec le cacao dans la plantation. Or au Cameroun, c'est une autre situation qui est en vogue dans ce pays où Bisseleua et al. (2008) ont montré que l'agroforesterie à base du Cacao est également confrontée à un problème de déprédation du fait des actions anthropiques et des attaques des insectes. Parmi les problèmes de l'agroforesterie à base d'anacardier au Bénin, l'aspect foncier ne peut pas être occulté. Aujourd'hui, certaines plantations de Anacardium occidentale sont prises d'assaut par les populations qui sont à la quête de l'espace pour construire leurs habitations (Photo 2). Ces espaces sont aussi lotis par les autorités communales à Savalou.

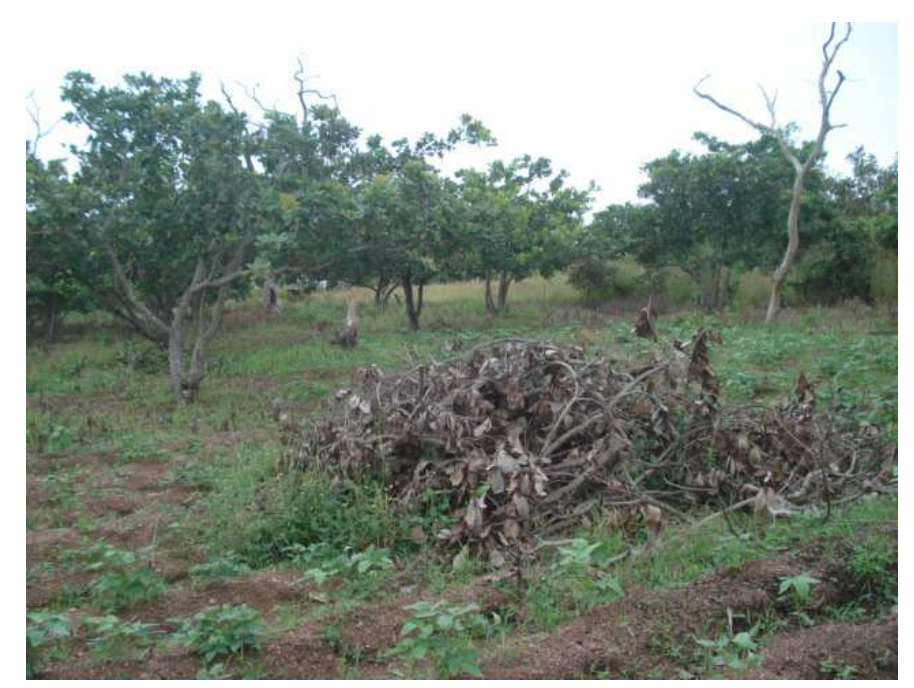

Photo 1: Déboisement dans une agro-forêt avec seulement quelques pieds de Pterocarpus erinaceus conservés. Cliché : Dadegnon, avril 2011. 


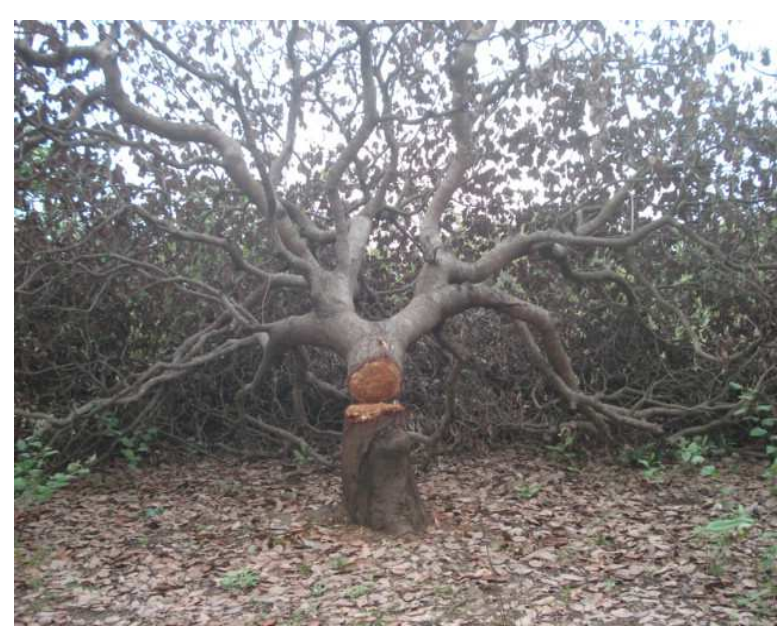

Photo 2: Un pied de-Anacardium occidentale abattu dans un champ pour des besoins de construction. Cliché : Dadegnon, avril 2011.

\section{Conclusion}

Au total, l'étude de la diversité biologique végétale au sein des agro-forêts à base d'anacardier révèle un potentiel non négligeable de taxon y compris même les plus menacés du Bénin. Ainsi, 38 espèces végétales appartenant à 19 familles sont inféodées aux plantations d'anacardier dans la commune de Savalou, ce qui illustre le caractère conservateur de la diversité végétale de ces écosystèmes. La présence d'espèces végétales menacées dans ces agro-forêts confirme leur utilité écologique et justifie la nécessité de leur conservation. Ces résultats montrent que l'agroforesterie peut être un outil puissant pour la conservation de la biodiversité végétale, contrairement à la culture itinérante habituellement pratiquée au Bénin. Par rapport à l'état des plantations d'anacardier dans la commune de Savalou, il est nécessaire d'opérer des aménagements pouvant faire de cette commune un pôle de développement économique et de la conservation de biodiversité.

Par ailleurs, l'agroforesterie à base d'anacardier peut contribuer à l'amélioration des conditions de vie des populations locales dans la mesure où elle favorise la souveraineté alimentaire des populations paysannes. Les systèmes agro-forestiers s'inscrivent donc dans le cadre du développement durable car ils visent concomitamment la conservation des espèces de valeur et le bien-être des populations locales.

\section{REMERCIEMENTS}

Les auteurs tiennent à exprimer leurs profonds remerciements aux évaluateurs dont la qualité et la pertinence des observations et suggestions ont permis d'améliorer significativement la qualité du manuscrit.

\section{REFERENCES}

Aïchéou DA. 2011. Production et commercialisation des fruits et légumes à Sèhouè dans la commune de Toffo. Mémoire de Maîtrise de Géographie, FLASH/UAC, 90 p

Akouègninou A. 2004. Recherches botaniques et écologiques sur les forêts actuelles au Bénin. Thèse d'Etat es Sciences. Université de Cocody Abijan, 326p.

Akoègninou A, Van der Burg WJ, Van der Maesen LJG. 2006. Flore Analytique du Bénin. Backhuys Publishers: Wageningen; 1034 p. 
Atchadé J-C. 2004. Etat des ressources zoogénétiques, Rapport national, République du Bénin, 71 p.

Bisseleua D, Herve B, Stefan V. 2008. Plant biodiversity and vegetation structure in traditional cocoa forest gardens in southern Cameroon un-der different management. Biodiversity Conserv, 17: 1821-1835.

Blondel J. 1975. L'analyse des peuplements d'oiseaux, éléments d'un diagnostic écologique I. la méthode des échantillonnages fréquentiels progressifs (E.F.P). Rev. Ecol, la Terre et la Vie, 29: 533-589.

Braun-Blanquet J. 1932 : Plant Sociology. The Study of Plant Communities. Me Gray Hill: New york, London; 439 p.

Daniele C, Michael K. 2011. Conservation Value of Cacao Agroforestry Systems for Terrestrial Herbaceous Speciesin Central Sulawesi, Indonesia. Biotropica, 43(6): 755-762.

Djèdji MT. 2011. Ethnobotanique et phytosociologie appliquée à l'aménagement des stations à Parkia biglobosa (Jacq). R. Br. ex G. don du Bénin : cas du Département de l'Atacora (Nord-Ouest Bénin). Mémoire de maîtrise de géographie, DGAT/FLASH/UAC, 130 p.

Djogbénou F. 2007. Impacts environnementaux des stratégies d'adaptation de l'agriculture à l'évolution du climat dans le département des collines, Mémoire de maîtrise de géographie, FLASH/UAC, 83 p.

Dussault CF. 2008. L'agroforesterie comme outil de développement durable dans les pays en voie de développement. Mémoire de maître en écologie internationale, Faculté des Sciences, Université de Sherbrooke, Québec, Canada, 106 p.

Lott JE, Howard SB, Ong, CK, Black CR. 2000. Long-term productivity of a
Grevillea robusta-based overstorey agroforestry system in semi-arid Kenya I. Tree growth. For. Ecol. \& Mgmt., 139: 175-186.

Moguel P, Toledo VM. 1999. Biodiversity Conservation in Traditional Coffee Systems of Mexico. Cons. Biol., 13: 1121.

Monra Jeremie LK. 2011. Pratiques de l'agroforterie dans la commune de bembéréke, mémoire de maîtrise de Géographie, UAC, 89 pages.

Okullo JBL, Waithum G. 2007. Diversity and conservation of on-farm woody plants by field types in Paromo Subcounty, Nebbi District, north-western Uganda. Af. J. Ecol. 45: 59-66.

Pielou EC. 1969. An Introduction to Mathematical Ecology. Wiley: New York.

Shannon CE. 1948. A mathematical theory of communications. Bell Syst. Techn. J., 27: 623656.

Soto-Pinto L, Perfecto I, Castillo-Hernandez J, Caballero-Nieto J. 2000. Shade effect on coffee production at the northern Tzeltal zone of the state of Chiapas, Mexico. Ag., Ecosyst. \& Env., 80: 91-69.

Tandjiékpon MA. 2005. Caractérisation du système agro-forestier à base de l'anacardier (Anacardium occidentale linnaeus) en zone de savane au Bénin, Mémoire de DEA, $122 \mathrm{p}$.

Wala K, Sinsin B, Guelly KA, Kokou K, Akpagana K. 2005. Typologie et structure des parcs agro-forestiers dans la préfecture de Doufelgou (Togo). Sécheresse, 16(3): 209-216.

Yabi I. 2004. Rôle de l'agroforesterie à base d'anacardier dans la dynamique de l'occupation du sol dans la région des monts Kouffé (secteur Agbassa-Idadjo), Mémoire de DEA, UAC/FLASH/DGAT, 77 p. + annexe. 
Yabi I. 2008. Etude de l'agroforesterie à base d'anacardier et des contraintes climatiques à son développement dans le centre du Bénin. Thèse de doctorat unique de géographie, EDP/FLASH, $\mathrm{UAC}, 240 \mathrm{p}$.

Yabi I, Boko M, Afouda F, Sinsin B. 2010. Vulnérabilité de l'agroforesterie à base de l'anacardier aux changements climatiques dans le centre du Bénin. Journal de la Recherche Scientifique de l'Université de Lomé (Togo), Série B, 12(2): 19-26.

Yabi I, Afouda F, Tohozin AY, Sinsin B, Boko M. 2012. Agroforesterie a base de l'anacardier et dynamique de l'occupation du sol sur l'axe Agbassa-Idajo (Région des monts Kouffè au Bénin). Revue de Géographie du Laboratoire de Recherche sur la Dynamique des Milieux et des Sociétés (LADYMES), 9: 91-103.

Zounmènou AG. 2007. Production, transport et commercialisation des produits vivriers en milieu rural : cas de la commune de Dangbo, Mémoire de Maîtrise de Géographie, FLASH/UAC, 80 p. 